\title{
Analytical Solutions for a General Mixed Initial-Boundary Value Problem Corresponding to Hydromagnetic Flows of Second Grade Fluids through Porous Medium
}

\author{
Constantin Fetecau ${ }^{1, *}$, Dumitru Vieru ${ }^{2}$ \\ ${ }^{1}$ Section of Mathematics, Academy of Romanian Scientists, 050094 Bucharest, Romania. \\ ${ }^{2}$ Department of Theoretical Mechanics, Technical University of Iasi, 700050 Iasi, Romania.
}

\begin{abstract}
How to cite this paper: Constantin Fetecau, Dumitru Vieru. (2021) Analytical Solutions for a General Mixed Initial-Boundary Value Problem Corresponding to Hydromagnetic Flows of Second Grade Fluids through Porous Medium. Journal of Applied Mathematics and Computation, 5(3), 225-236.

DOI: $10.26855 /$ jamc.2021.09.009
\end{abstract}

Received: August 16, 2021

Accepted: September 8, 2021

Published: September 24, 2021

*Corresponding author: Constantin Fetecau, Section of Mathematics, Academy of Romanian Scientists, 050094 Bucharest, Romania.

Email: c_fetecau@yahoo.com

\begin{abstract}
A general mixed initial-boundary value problem describing hydro magnetic flows of the incompressible second grade fluids (ISGF) between infinite horizontal parallel plates embedded in a porous medium is analytically studied. The fluid motion is induced by the lower plate that applies an arbitrary time-dependent shear stress to the fluid. Closed form expressions are established for the dimensionless velocity field and the volume flux per unit width of a plane normal to the flow direction. They can generate exact solutions for any flow of this type of ISGF and the problem in discussion is completely solved. For illustration, three motions with technical relevance are taken into consideration and the necessary time to touch the steady or permanent state for two motions which become steady in time is graphically determined. In addition, it is proved that the solutions corresponding to motions induced by ramp-type shear stresses on the boundary can be easily determined if the similar solutions for the motion generated by a constant shear stress on the boundary are known.
\end{abstract}

\section{Keywords}

Second Grade Fluids, Hydromagnetic Flows, Porous Medium, Parallel Plates, General Solutions

\section{Introduction}

Fluids' motion between parallel plates, as well as their flow over an infinite plate, has multiple applications in engineering and the science in general. It can be generated, for instance, by one of plates that move in its plane or apply a shear stress to the fluid. If the fluid was at rest at the initial moment, its motion may become steady or remains unsteady. For steady motions the experimentalists are interested to know the required time to reach the steady-state. This is the time after which the fluid motion is characterized by the steady or permanent solutions. Main purpose of this work is to bring to light the influence of magnetic field and the porous medium on the need time to touch the steady state for some motions of the ISGF between two infinite horizontal parallel plates. For completion and a larger applicability, an arbitrary time-dependent shear stress will be applied on the boundary.

In the existing literature there are many exact solutions for isothermal unsteady motions of incompressible Newtonian fluids between parallel plates. Some of them can be found in the book of Schlichting [1] and the review article of Wang [2]. Interesting exact solutions for the unsteady Couette flow of same fluids have been obtained by Erdogan [3, 4] and 
Mendiburu et al. [5]. The magneto hydrodynamic (MHD) Couette flow was also studied due to its multiple applications in hydrology and engineering structures. Interaction between the magnetic field and the electrical conducting fluid produces effects with important applications in physics, chemistry, hydrology, geothermal systems etc. The steady MHD Couette flow of Newtonian fluids was investigated by Singha and Choudhary [6] and Kiema et al. [7] using the Laplace, respectively the Sumudu transform. A numerical study of the unsteady hydro magnetic Couette flow has been provided by Onyango et al. [8].

On the other hand, the flows of fluids through porous media have important applications in the agricultural engineering, petroleum industries and oil reservoir technology as well as in geophysical and astrophysical studies. The flow of incompressible Newtonian fluids through horizontal channels of porous media has been numerically studied by Al-Hadhrami et al. [9] while the MHD flow due to sinusoidal pressure gradient were investigated by Dash and Ojha [10]. Recently, Fetecau and Narahary [11] and Javaid et al. [12] established general solutions for MHD motions of the incompressible Newtonian fluids between infinite horizontal parallel plates through a porous medium when the lower plate moves in its plane with an arbitrary velocity or applies a time-dependent shear stress to the fluid. Unfortunately, similar solutions for unsteady motions of non-Newtonian fluids are lacking in the literature.

First exact solutions for the velocity field corresponding to an unsteady motion of non-Newtonian fluids, more exactly second grade fluids, between parallel plates seem to be those of Ting [13]. Much later Rajagopal [14] provided the steady-state velocity field for the motion induced by cosine oscillations of the lower plate. Steady solutions for unsteady MHD flows of the same fluids through a porous medium have been obtained by Hayat et al. [15] when one of plates moves in its plane and the other one being stationary or free surface. General solutions for the simple Couette flow of these fluids when the lower plate applies a time-dependent shear stress to the fluid have been obtained by Imran et al. [16]. Recently, the MHD free convection flow of second grade fluids between parallel plates were studied by Ali et al. [17] in the presence of a porous medium when the upper plate exponentially moves in its plane.

In the present work, general solutions for the isothermal unsteady MHD flow of ISGF between infinite horizontal parallel plates embedded in a porous medium are determined. The fluid motion is generated by the lower plate that applies an arbitrary time-dependent shear stress to the fluid and the problem in consideration is completely solved. For illustration, three special cases are considered and interesting results are obtained. Solutions corresponding to motions due to ramp-type shear stresses on the boundary are completely determined if the similar solutions for the motion induced by a constant shear stress are known. The required time to touch the steady state for motions produced by constant or oscillatory shear stresses on the boundary, which become steady in time, is graphically determined. It was found that the steady state is rather obtained in the presence of a magnetic field or porous medium.

\section{Governing equations}

Let us assume that an electrically conducting ISGF is at rest between two infinite horizontal parallel plates at the distance $h$ apart embedded in a porous medium. Constitutive equation of such a fluid is given by the following relation [18]

$$
\boldsymbol{T}=-p \boldsymbol{I}+\mu \mathbf{A}_{1}+\alpha_{1} \boldsymbol{A}_{2}+\alpha_{2} \boldsymbol{A}_{1}^{2},
$$

where $\boldsymbol{T}$ is the Cauchy stress tensor, $p$ is the hydrostatic pressure, $\boldsymbol{I}$ the identity tensor, $\boldsymbol{\mu}$ is the fluid viscosity, $\alpha_{1}$ and $\alpha_{2}$ are material constants and

$$
\boldsymbol{A}_{1}=\boldsymbol{L}+\boldsymbol{L}^{T}, \quad \boldsymbol{A}_{2}=\frac{d \boldsymbol{A}_{1}}{d t}+\boldsymbol{A}_{1} \boldsymbol{L}+\boldsymbol{L}^{T} \boldsymbol{A}_{1},
$$

are the first two Rivlin-Ericksen tensors. In the above relation $\boldsymbol{L}$ is the gradient of the velocity vector $\boldsymbol{V}$ and $d / d t$ denotes the material time derivative. The assumption that the Helmholtz free energy is minimum in equilibrium and the Clausius-Duhem inequality impose the following restrictions [19]

$$
\alpha_{1} \geq 0 \text {, respectively } \alpha_{1}+\alpha_{2}=0 \text {, }
$$

for the two material constants $\alpha_{1}$ and $\alpha_{2}$.

We also assume that a magnetic field of strength $\mathrm{B}$ acts perpendicular to the plates and the magnetic Reynolds number of the fluid is small enough. This last assumption, which is valid at least for partially ionized fluids or metallic liquids [20], allows us to neglect the induced magnetic field. At moderate values of the magnetic parameter, the Hall effects do not have a meaningful influence on velocity and shear stress profiles and can be also neglected. At the moment $t=0^{+}$, the lower plate begins to apply a time-dependent shear stress $-S f(t)$ to the fluid. Here, the non-dimensional function $f(\cdot)$ is piecewise continuous and $f(0)=0$ while $S$ is a constant shear stress. Owing to the shear the fluid begins to move and, because the plates are boundless, we are looking for a velocity field of the form 


$$
\boldsymbol{v}=\boldsymbol{V}(y, t)=u(y, t) \mathbf{i},
$$

where $\boldsymbol{i}$ is the unit vector lengthways the $x$-axis of a convenient Cartesian coordinate system $x, y$ and $z$ having the $y$-axis perpendicular to the plates.

Introducing the velocity $\boldsymbol{v}(y, t)$ from Eq. (4) in (1), it results that the non-trivial shear stress $\tau(y, t)=T_{x y}(y, t)$ is given by the relation

$$
\tau(y, t)=\mu\left(1+\alpha_{1} \frac{\partial}{\partial t}\right) \frac{\partial u(y, t)}{\partial y} ; \quad 0<y<h, t>0 .
$$

The incompressibility condition is identically satisfied while the momentum balance, in the absence of a pressure gradient in the flow direction, reduces to the following relevant partial differential equation [15]

$$
\rho \frac{\partial u(y, t)}{\partial t}=\frac{\partial \tau(y, t)}{\partial y}-\sigma B^{2} u(y, t)-\frac{\mu \phi}{k}\left(1+\alpha_{1} \frac{\partial}{\partial t}\right) u(y, t) ; \quad 0<y<h, t>0,
$$

where $\rho$ is the fluid density, $\sigma$ is the electrical conductivity, $\phi(0<\phi<1)$ is the porosity and $k>0$ is the permeability of the porous medium.

Substituting $\tau(y, t)$ from Eq. (5) in (6), the following partial differential equation

$$
\rho \frac{\partial u(y, t)}{\partial t}=\mu\left(1+\alpha_{1} \frac{\partial}{\partial t}\right) \frac{\partial^{2} u(y, t)}{\partial y^{2}}-\sigma B^{2} u(y, t)-\frac{\mu \phi}{k}\left(1+\alpha_{1} \frac{\partial}{\partial t}\right) u(y, t) ; \quad 0<y<h, t>0,
$$

is obtained for the dimensional velocity field $u(y, t)$. The corresponding initial and boundary conditions are given by the following relations

$$
\begin{gathered}
u(y, 0)=0 ; \quad 0 \leq y \leq h, \\
\tau(0, t)=\left.\mu\left(1+\alpha_{1} \frac{\partial}{\partial t}\right) \frac{\partial u(y, t)}{\partial y}\right|_{y=0}=-S f(t), \quad u(h, t)=0 ; \quad t>0 .
\end{gathered}
$$

The volume flux across a plane normal to the flow and per unit width of this plane is

$$
Q(t)=\int_{0}^{h} u(y, t) d y \text {. }
$$

Our interest here is to determine exact expressions for the dimensionless velocity field and the corresponding volume flux. The velocity field will be used to determine the required time to reach the steady state for motions which become steady (permanent) in time. In order to do that the following non-dimensional variables, functions and parameters

$$
y^{*}=\frac{y}{h}, t^{*}=\frac{S}{\mu} t, u^{*}=\frac{\mu}{h S} u, \tau^{*}=\frac{\tau}{S}, \alpha^{*}=\frac{\alpha_{1} S}{\mu}, f^{*}\left(t^{*}\right)=f\left(\frac{\mu}{S} t^{*}\right), Q^{*}=\frac{\mu}{S h^{2}} Q,
$$

are taken into consideration. Introducing these dimensionless entities in the equalities (7)-(9) and renouncing to the star notation, the following mixed initial-boundary value problem

$$
\begin{gathered}
\operatorname{Re} \frac{\partial u(y, t)}{\partial t}=\left(1+\alpha \frac{\partial}{\partial t}\right) \frac{\partial^{2} u(y, t)}{\partial y^{2}}-M u(y, t)-K\left(1+\alpha \frac{\partial}{\partial t}\right) u(y, t) ; \quad 0<y<1, t>0, \\
u(y, 0)=0 ; \quad 0 \leq y \leq 1, \\
\left.\left(1+\alpha \frac{\partial}{\partial t}\right) \frac{\partial u(y, t)}{\partial y}\right|_{y=0}=-f(t), \quad u(1, t)=0 ; \quad t>0,
\end{gathered}
$$

is obtained for the dimensionless velocity field $u(y, t)$. The non-dimensional constants $\operatorname{Re}, M$ and $K$ defined by the next relations

$$
\operatorname{Re}=\frac{V h}{v}, \quad M=\frac{\sigma B^{2}}{\mu} h^{2} \text { and } K=\frac{\phi}{k} h^{2} \text {, }
$$


where $V=S h / \mu$ is a characteristic velocity, are Reynolds number, magnetic parameter and the porosity parameter, respectively.

As soon as the dimensionless velocity field $u(y, t)$ is known, the corresponding shear stress $\tau(y, t)$ and the volume flux $Q(t)$ per unit width of a plane perpendicular to the flow are immediately determined using the relationships

$$
\tau(y, t)=\left(1+\alpha \frac{\partial}{\partial t}\right) \frac{\partial u(y, t)}{\partial y}, \quad Q(t)=\int_{0}^{1} u(y, t) d y .
$$

In the following, we shall solve the mixed initial-boundary value problem (12)-(14) using the finite Fourier cosine transform defined by the equality (A1) from Appendix.

\section{Solution of the problem}

Multiplying Eq. (12) by $\cos [(2 n+1) \pi / 2]$, integrating the result with regard to $y$ from zero to one and taking into account the equality (A2) from Appendix, it results that the finite Fourier cosine transform $u_{F n}(t)$ of $u(y, t)$ has to satisfy the ordinary differential equation

$$
\left[\operatorname{Re}+\alpha\left(\mu_{n}^{2}+K\right)\right] \frac{d u_{F n}(t)}{d t}+\left(\mu_{n}^{2}+K_{e f f}\right) u_{F n}(t)=f(t) ; t>0 \text { and } n=0,1,2,3, \ldots,
$$

where $\mu_{n}=(2 n+1) \pi / 2$ and $K_{\text {eff }}=K+M$. The next initial conditions

$$
u_{F n}(0)=0 \text { for } n=0,1,2,3, \ldots \text {, }
$$

have to be also satisfied.

The solution of the ordinary differential equation (17) with the initial condition (18) is

$$
u_{F n}(t)=\frac{1}{\operatorname{Re}+\alpha\left(\mu_{n}^{2}+K\right)} \int_{0}^{t} f(t-s) \exp \left[-\frac{\mu_{n}^{2}+K_{\text {eff }}}{\operatorname{Re}+\alpha\left(\mu_{n}^{2}+K\right)} s\right] d s
$$

Consequently, the non-dimensional velocity $u(y, t)$ corresponding to this motion has the form

$$
u(y, t)=2 \sum_{n=0}^{\infty} \frac{\cos \left(\mu_{n} y\right)}{\operatorname{Re}+\alpha\left(\mu_{n}^{2}+K\right)} \int_{0}^{t} f(t-s) \exp \left[-\frac{\mu_{n}^{2}+K_{e f f}}{\operatorname{Re}+\alpha\left(\mu_{n}^{2}+K\right)} s\right] d s .
$$

In this form, the velocity field $u(y, t)$ satisfies the initial condition (13) but the boundary condition $(14)_{2}$ seems to be unsatisfied. However, using the equality (A3) from Appendix, $u(y, t)$ can be also written in the following equivalent form

$$
u(y, t)=(1-y) f(t)-2 K_{e f f} f(t) \sum_{n=0}^{\infty} \frac{\cos \left(\mu_{n} y\right)}{\mu_{n}^{2}\left(\mu_{n}^{2}+K_{e f f}\right)}-2 \sum_{n=0}^{\infty} \frac{\cos \left(\mu_{n} y\right)}{\mu_{n}^{2}+K_{e f f}} \int_{0}^{t} f^{\prime}(t-s) \exp \left[-\frac{\mu_{n}^{2}+K_{e f f}}{\operatorname{Re}+\alpha\left(\mu_{n}^{2}+K\right)} s\right] d s,
$$

which clearly satisfies the initial condition (13) and the boundary condition $(14)_{2}$. A new equivalent form of the dimensionless velocity field $u(y, t)$, which satisfy all imposed initial and boundary conditions, is given by the following relation

$$
u(y, t)=\frac{1-y}{\alpha} \int_{0}^{t} f(t-s) \exp \left(-\frac{s}{\alpha}\right) d s+\frac{2}{\alpha} \sum_{n=0}^{\infty} \frac{\cos \left(\mu_{n} y\right)}{a_{n} \mu_{n}^{2}} \int_{0}^{t} f(t-s)\left\{\alpha \mu_{n}^{2} \exp \left[-\frac{\mu_{n}^{2}+K_{e f f}}{a_{n}} s\right]-a_{n} \exp \left(-\frac{s}{\alpha}\right)\right\} d s
$$

where $a_{n}=\operatorname{Re}+\alpha\left(\mu_{n}^{2}+K\right)$.

The corresponding shear stress $\tau(y, t)$ and the dimensionless frictional forces per unit area exerted by the fluid on the plates are immediately determined using Eqs. (16) ${ }_{1}$ and (20) or (21). The non-dimensional volume flux per unit width of a plane normal to the flow direction is given by the following relation

$$
Q(t)=\frac{1}{2} f(t)-2 K_{e f f} f(t) \sum_{n=0}^{\infty} \frac{(-1)^{n}}{\mu_{n}^{3}\left(\mu_{n}^{2}+K_{e f f}\right)}-2 \sum_{n=0}^{\infty} \frac{(-1)^{n}}{\mu_{n}\left(\mu_{n}^{2}+K_{e f f}\right)} \int_{0}^{t} f^{\prime}(t-s) \exp \left[-\frac{\mu_{n}^{2}+K_{e f f}}{\operatorname{Re}+\alpha\left(\mu_{n}^{2}+K\right)} s\right] d s .
$$


In the absence of magnetic or porous effects, the corresponding solutions can be obtained taking $M=0$, respectively $K=0$ in the previous relations. In the absence of both porous and magnetic effects, when $K=K_{\text {eff }}=0$, the relations (21) and (22) take the simpler forms

$$
\begin{gathered}
u(y, t)=(1-y) f(t)-2 \sum_{n=0}^{\infty} \frac{\cos \left(\mu_{n} y\right)}{\mu_{n}^{2}} \int_{0}^{t} f^{\prime}(t-s) \exp \left[-\frac{\mu_{n}^{2}}{\operatorname{Re}+\alpha \mu_{n}^{2}} s\right] d s, \\
Q(t)=\frac{1}{2} f(t)-2 \sum_{n=0}^{\infty} \frac{(-1)^{n}}{\mu_{n}^{3}} \int_{0}^{t} f^{\prime}(t-s) \exp \left[-\frac{\mu_{n}^{2}}{\operatorname{Re}+\alpha \mu_{n}^{2}} s\right] d s .
\end{gathered}
$$

Finally, taking $\alpha=0$ into above relations, similar solutions corresponding to incompressible Newtonian fluids performing the same motion are recovered (see Eqs. (17) and (20) of Javaid et al. [12]). These solutions as well as those corresponding to the Stokes' problems [21] do not depend of the magnetic and porous parameters $M$ and $K$ independently, but only by a combination of them $K_{\text {eff }}=M+K$ that was called the effective permeability.

\section{Some special cases with engineering applications}

As the function $f(\cdot)$ is arbitrary, the relations (21) and (22) can generate exact solutions for any motion of this type of the ISGF. Consequently, the problem of MHD flow of incompressible second grade fluids between infinite horizontal parallel plates embedded in a porous medium is completely solved when lower plate induces a shear stress to the fluid. In the following, for illustration as well as to get some physical insight of results that have been obtained, three flows with technical relevance are considered.

\subsection{Case $f(t)=H(t)$ (Flow due to constant shear stress on the boundary)}

By replacing $f(\cdot)$ with the Heaviside unit step function $H(\cdot)$ in Eqs. (21) and (22), the dimensionless velocity field (see also Eq. (A4) from Appendix)

$$
u_{S}(y, t)=1-y-2 K_{\text {eff }} \sum_{n=0}^{\infty} \frac{\cos \left(\mu_{n} y\right)}{\mu_{n}^{2}\left(\mu_{n}^{2}+K_{\text {eff }}\right)}-2 \sum_{n=0}^{\infty} \frac{\cos \left(\mu_{n} y\right)}{\mu_{n}^{2}+K_{\text {eff }}} \exp \left[-\frac{\mu_{n}^{2}+K_{\text {eff }}}{\operatorname{Re}+\alpha\left(\mu_{n}^{2}+K\right)} t\right] ; t>0
$$

and the adequate volume flux per unit width of a plane normal to the flow

$$
Q_{S}(t)=\frac{1}{2}-2 K_{\text {eff }} \sum_{n=0}^{\infty} \frac{(-1)^{n}}{\mu_{n}^{3}\left(\mu_{n}^{2}+K_{\text {eff }}\right)}-2 \sum_{n=0}^{\infty} \frac{(-1)^{n}}{\mu_{n}\left(\mu_{n}^{2}+K_{\text {eff }}\right)} \exp \left[-\frac{\mu_{n}^{2}+K_{\text {eff }}}{\operatorname{Re}+\alpha\left(\mu_{n}^{2}+K\right)} t\right] ; t>0,
$$

corresponding to the motion due to a constant shear stress $S$ on the boundary are obtained. In the absence of magnetic and porous effects, the relations (25) and (26) are in agreement with those obtained by Imran et al. [16, Eqs. (23) and (24)] using a different technique. The sign difference is due to the boundary conditions which are taken with opposite signs. For $t=0$, as expected, the volume flux $Q(0)$ becomes zero (see Eq. (A5) from Appendix).

Of course, this motion becomes steady or permanent in time and making $t \rightarrow \infty$ in Eqs. (25) and (26), the steady or permanent solutions

$$
u_{N S p}(y)=1-y-2 K_{e f f} \sum_{n=0}^{\infty} \frac{\cos \left(\mu_{n} y\right)}{\mu_{n}^{2}\left(\mu_{n}^{2}+K_{\text {eff }}\right)}, \quad Q_{N S p}=\frac{1}{2}-2 K_{\text {eff }} \sum_{n=0}^{\infty} \frac{(-1)^{n}}{\mu_{n}^{3}\left(\mu_{n}^{2}+K_{\text {eff }}\right)},
$$

corresponding to Newtonian fluids performing the same motion are recovered [12, Eqs. $(23)_{1}$ and $(26)_{2}$ ]. This is not a surprise because the governing equations corresponding to steady motions of the incompressible Newtonian or second grade fluids are identical. Direct computations show that $u_{N S p}(y)$ and $Q_{N S p}$ can be presented in the simpler forms, namely

$$
u_{N S p}(y)=\frac{1}{\sqrt{K_{\text {eff }}}} \frac{\sinh \left[(1-y) \sqrt{K_{\text {eff }}}\right]}{\cosh \left(\sqrt{K_{\text {eff }}}\right)}, \quad Q_{N S p}=\frac{1}{K_{\text {eff }}} \frac{\cosh \left(\sqrt{K_{\text {eff }}}\right)-1}{\cosh \left(\sqrt{K_{\text {eff }}}\right)} .
$$

The equivalence of the solutions given by Eqs. (27) and (28) is graphically proved in Figure 1.

The corresponding shear stress $\tau_{N S p}(y)$ can be also presented in equivalent forms, i.e. 


$$
\tau_{N S p}(y)=-1+2 K_{\text {eff }} \sum_{n=0}^{\infty} \frac{\sin \left(\mu_{n} y\right)}{\mu_{n}\left(\mu_{n}^{2}+K_{\text {eff }}\right)} \text { or } \tau_{N S p}(y)=-\frac{\cosh \left[(1-y) \sqrt{K_{\text {eff }}}\right]}{\cosh \left(\sqrt{K_{\text {eff }}}\right)},
$$

while the dimensionless frictional force per unit area exerted by the fluid on the stationary plate is given by any one of the expressions

$$
\tau_{N S p}(1)=-1+2 K_{\text {eff }} \sum_{n=0}^{\infty} \frac{(-1)^{n}}{\mu_{n}\left(\mu_{n}^{2}+K_{e f f}\right)} \quad \text { or } \quad \tau_{N S p}(1)=-\frac{1}{\cosh \left(\sqrt{K_{e f f}}\right)} .
$$

In conclusion, the steady components of the dimensionless velocity field, volume flux and non-trivial shear stress corresponding to this motion of ISGF do not depend on porous and magnetic parameters $K$ and $M$ independently, but by a combination of them $K_{\text {eff }}=K+M$ which in the literature was called effective permeability [11]. Consequently, a two parameter approach for their graphical representations is superfluous or even misleading.

\subsection{Case $f(t)=t^{\beta}(\beta>0)$ (Flow induced by a ramp-type shear stress on the boundary)}

Dimensionless velocity field and the adequate volume flux corresponding to this case, namely

$$
\begin{aligned}
& u_{\beta}(y, t)=(1-y) t^{\beta}-2 K_{e f f} t^{\beta} \sum_{n=0}^{\infty} \frac{\cos \left(\mu_{n} y\right)}{\mu_{n}^{2}\left(\mu_{n}^{2}+K_{e f f}\right)}-2 \beta \sum_{n=0}^{\infty} \frac{\cos \left(\mu_{n} y\right)}{\mu_{n}^{2}+K_{e f f}^{t}} \int_{0}^{t}(t-s)^{\beta-1} \exp \left[-\frac{\mu_{n}^{2}+K_{e f f}}{\operatorname{Re}+\alpha\left(\mu_{n}^{2}+K\right)} s\right] d s ; t>0, \\
& Q_{\beta}(t)=\frac{1}{2} t^{\beta}-2 K_{e f f} t^{\beta} \sum_{n=0}^{\infty} \frac{(-1)^{n}}{\mu_{n}^{3}\left(\mu_{n}^{2}+K_{e f f}\right)}-2 \beta \sum_{n=0}^{\infty} \frac{(-1)^{n}}{\mu_{n}\left(\mu_{n}^{2}+K_{e f f}\right)} \int_{0}^{t}(t-s)^{\beta-1} \exp \left[-\frac{\mu_{n}^{2}+K_{e f f}}{\operatorname{Re}+\alpha\left(\mu_{n}^{2}+K\right)} s\right] d s ; t>0,
\end{aligned}
$$

are immediately obtained substituting $f(t)$ by $t^{\beta}$ in Eqs. (21) and (22).

It is worth to point out the fact that the solutions corresponding to $\beta=1$, namely

$$
\begin{gathered}
u_{1}(y, t)=(1-y) t-2 K_{e f f} t \sum_{n=0}^{\infty} \frac{\cos \left(\mu_{n} y\right)}{\mu_{n}^{2}\left(\mu_{n}^{2}+K_{e f f}\right)}-2 \sum_{n=0}^{\infty} \frac{\left[\operatorname{Re}+\alpha\left(\mu_{n}^{2}+K\right)\right] \cos \left(\mu_{n} y\right)}{\left(\mu_{n}^{2}+K_{e f f}\right)^{2}}\left[1-\exp \left(-\frac{\mu_{n}^{2}+K_{e f f}}{\operatorname{Re}+\alpha\left(\mu_{n}^{2}+K\right)} t\right) ; t>0,\right. \\
Q_{1}(t)=\frac{1}{2} t-2 K_{e f f} t \sum_{n=0}^{\infty} \frac{(-1)^{n}}{\mu_{n}^{3}\left(\mu_{n}^{2}+K_{e f f}\right)}-2 \sum_{n=0}^{\infty} \frac{(-1)^{n}\left[\operatorname{Re}+\alpha\left(\mu_{n}^{2}+K\right)\right]}{\mu_{n}\left(\mu_{n}^{2}+K_{e f f}\right)}\left[1-\exp \left(-\frac{\mu_{n}^{2}+K_{e f f}}{\operatorname{Re}+\alpha\left(\mu_{n}^{2}+K\right)} t\right)\right] t>0,
\end{gathered}
$$

can be written as simple integrals from $u_{S}(y, t)$, respectively $Q_{S}(t)$, i.e.

$$
u_{1}(y, t)=\int_{0}^{t} u_{s}(y, s) d s, \quad Q_{1}(t)=\int_{0}^{t} Q_{S}(s) d s .
$$

Moreover, following the same way as in [22], one can prove that

$$
u_{\beta}(y, t)=\Gamma(\beta+1) I_{t}^{\beta}\left\{u_{S}(y, t)\right\}, \quad Q_{\beta}(t)=\Gamma(\beta+1) I_{t}^{\beta}\left\{Q_{S}(t)\right\},
$$

where the Rieman-Liouville fractional operator $I_{t}^{\beta}\{\cdot\}$ is defined by following relation

$$
I_{t}^{\beta}\{h(t)\}=\frac{1}{\Gamma(\beta)} \int_{0}^{t}(t-s)^{\beta-1} h(s) d s
$$

and $\Gamma(\cdot)$ is the Gamma function. If $\beta=n$ (a natural number), the equalities (36) take the simpler forms

$$
\begin{gathered}
u_{n}(y, t)=(n) ! \int_{0}^{t} \int_{0}^{s_{1}} \int_{0}^{s_{2}} \ldots \int_{0}^{s_{n-1}} u_{S}\left(y, s_{n}\right) d s_{n} d s_{n-1} \ldots d s_{1}, \\
Q_{n}(t)=(n) ! \int_{0}^{t} \int_{0}^{s_{1}} \int_{0}^{s_{2}} \ldots \int_{0}^{s_{n-1}} Q_{S}\left(s_{n}\right) d s_{n} d s_{n-1} \ldots d s_{1} .
\end{gathered}
$$

Of course, similar relations are also valid between corresponding shear stresses $\tau_{\beta}(y, t)$ and $\tau_{S}(y, t)$. Consequently, the solutions corresponding to motions generated by the lower plate that applies shear stresses of the form $S t^{\beta}$ to the 
fluid can be determined if the adequate solutions for the motion induced by a constant shear stress $S$ on the boundary are known.

\subsection{Case $f(t)=H(t) \cos (\omega t)$ or $H(t) \sin (\omega t)$ (Flows due to oscillatory shear stresses)}

By replacing the function $f(t)$ with $H(t) \cos (\omega t)$ or $H(t) \sin (\omega t)$ in Eq. (21) and again using Eq. (A3) from Appendix, it is not difficult to show that the dimensionless starting velocities $u_{c}(y, t)$ and $u_{s}(y, t)$ corresponding to motions due to cosine, respectively sine oscillations of the shear stress on the boundary can be presented as sum of steady-state (permanent or long time) and transient components. More exactly they can be written under forms

where

$$
u_{c}(y, t)=u_{c p}(y, t)+u_{c t}(y, t), \quad u_{s}(y, t)=u_{s p}(y, t)+u_{s t}(y, t) ; 0<y<1, t>0,
$$

$$
\begin{aligned}
& u_{c p}(y, t)=(1-y) \cos (\omega t)+2 \omega \sin (\omega t) \sum_{n=0}^{\infty} \frac{\left[\operatorname{Re}+\alpha\left(\mu_{n}^{2}+K\right)\right] \cos \left(\mu_{n} y\right)}{\left(\mu_{n}^{2}+K_{e f f}\right)^{2}+\omega^{2}\left[\operatorname{Re}+\alpha\left(\mu_{n}^{2}+K\right)\right]^{2}} \\
& -2 \cos (\omega t) \sum_{n=0}^{\infty} \frac{K_{e f f}\left(\mu_{n}^{2}+K_{e f f}\right)+\omega^{2}\left[\operatorname{Re}+\alpha\left(\mu_{n}^{2}+K\right)\right]^{2}}{\mu_{n}^{2}\left\{\left(\mu_{n}^{2}+K_{\text {eff }}\right)^{2}+\omega^{2}\left[\operatorname{Re}+\alpha\left(\mu_{n}^{2}+K\right)\right]^{2}\right\}} \cos \left(\mu_{n} y\right), \\
& u_{c t}(y, t)=-2 \sum_{n=0}^{\infty} \frac{\left(\mu_{n}^{2}+K_{e f f}\right) \cos \left(\mu_{n} y\right)}{\left(\mu_{n}^{2}+K_{e f f}\right)^{2}+\omega^{2}\left[\operatorname{Re}+\alpha\left(\mu_{n}^{2}+K\right)\right]^{2}} \exp \left(-\frac{\mu_{n}^{2}+K_{e f f}}{\operatorname{Re}+\alpha\left(\mu_{n}^{2}+K\right)} t\right), \\
& u_{s p}(y, t)=(1-y) \sin (\omega t)-2 \omega \cos (\omega t) \sum_{n=0}^{\infty} \frac{\left[\operatorname{Re}+\alpha\left(\mu_{n}^{2}+K\right)\right] \cos \left(\mu_{n} y\right)}{\left(\mu_{n}^{2}+K_{e f f}\right)^{2}+\omega^{2}\left[\operatorname{Re}+\alpha\left(\mu_{n}^{2}+K\right)\right]^{2}} \\
& -2 \sin (\omega t) \sum_{n=0}^{\infty} \frac{K_{e f f}\left(\mu_{n}^{2}+K_{e f f}\right)+\omega^{2}\left[\operatorname{Re}+\alpha\left(\mu_{n}^{2}+K\right)\right]^{2}}{\mu_{n}^{2}\left\{\left(\mu_{n}^{2}+K_{e f f}\right)^{2}+\omega^{2}\left[\operatorname{Re}+\alpha\left(\mu_{n}^{2}+K\right)\right]^{2}\right\}} \cos \left(\mu_{n} y\right), \\
& u_{s t}(y, t)=2 \omega \sum_{n=0}^{\infty} \frac{\left[\operatorname{Re}+\alpha\left(\mu_{n}^{2}+K\right)\right] \cos \left(\mu_{n} y\right)}{\left(\mu_{n}^{2}+K_{e f f}\right)^{2}+\omega^{2}\left[\operatorname{Re}+\alpha\left(\mu_{n}^{2}+K\right)\right]^{2}} \exp \left(-\frac{\mu_{n}^{2}+K_{e f f}}{\operatorname{Re}+\alpha\left(\mu_{n}^{2}+K\right)} t\right) .
\end{aligned}
$$

Equivalent forms for the steady-state components $u_{c p}(y, t)$ and $u_{s p}(y, t)$, namely

$$
u_{c p}(y, t)=\mathfrak{R e}\left\{\frac{1}{1+i \omega \alpha} \frac{\sinh [\gamma(1-y)]}{\gamma \cosh (\gamma)} \mathrm{e}^{i \omega t}\right\}, u_{s p}(y, t)=\operatorname{Im}\left\{\frac{1}{1+i \omega \alpha} \frac{\sinh [\gamma(1-y)]}{\gamma \cosh (\gamma)} \mathrm{e}^{i \omega t}\right\},
$$

can be also obtained using the complex velocity $u_{p}(y, t)=u_{c p}(y, t)+i u_{s p}(y, t)$. In the above relations $\gamma=\sqrt{K_{\text {eff }}+i \omega(\operatorname{Re}+\alpha K)}$ and the equivalence of the solutions given by Eqs. (41) and (43) with those from (45) 1 , respectively $(45)_{2}$ is graphically proved by Figures $2 \mathrm{a}$ and $2 \mathrm{~b}$.

The corresponding components $\tau_{c p}(y, t)$ and $\tau_{s p}(y, t)$ of the dimensionless non-trivial shear stresses $\tau_{c}(y, t)$, respectively $\tau_{s}(y, t)$ are also given by the simple relations

$$
\tau_{c p}(y, t)=-\mathfrak{R e}\left\{\frac{\cosh [\gamma(1-y)]}{\cosh (\gamma)} \mathrm{e}^{i \omega t}\right\}, \tau_{s p}(y, t)=-\operatorname{Im}\left\{\frac{\cosh [\gamma(1-y)]}{\cosh (\gamma)} \mathrm{e}^{i \omega t}\right\},
$$

which clearly satisfy the boundary condition $(9)_{1}$ in non-dimensional form. Finally, it is worth pointing out the fact that making $\omega=0$ in Eqs. (40) 1 and (45) $)_{1}$, the solutions $u_{S}(y, t)$ and $u_{N S p}(y)$ (from the equalities (25), respectively (28) corresponding to the motion generated by a constant shear stress $S$ on the boundary are recovered. Finally, we recall that $\omega$ in the previous relations is the dimensionless frequency of the oscillations.

\section{Some numerical results and conclusions}

MHD isothermal unsteady flows of the ISGF between infinite horizontal parallel plates are analytically studied when porous effects are taken into consideration. The fluid motion, contrary to what is usually assumed in the existing literature, is generated by an arbitrary shear stress on the boundary. Actually, how the boundary moves is a consequence of the 
applied force and in Newtonian mechanics force is the cause and kinematics is the effect (for a detailed discussion on this problem see [23]). Exact general expressions are established for the dimensionless velocity field and the corresponding volume flux per unit width of a plane perpendicular to the flow direction. These expressions, obtained in a simple way using the finite Fourier cosine transform only, can be easily reduced to the similar solutions for Newtonian fluids performing the same motions.

Generally, such motions can become steady in time or remain non-steady. In the first case, in practice it is necessary to know the required time to reach the steady or permanent state. This is the time after which the fluid behavior can be characterized by the steady solutions. From mathematical point of view, it is the time after which the diagrams of starting solutions superpose over those of the steady-state solutions. This is the reason that the starting solutions corresponding to such motions have been presented as sum of their steady state and transient components. For the validation of obtained results, the steady solutions have been presented in different forms whose equivalence was graphically proved by Figures 1 and 2.
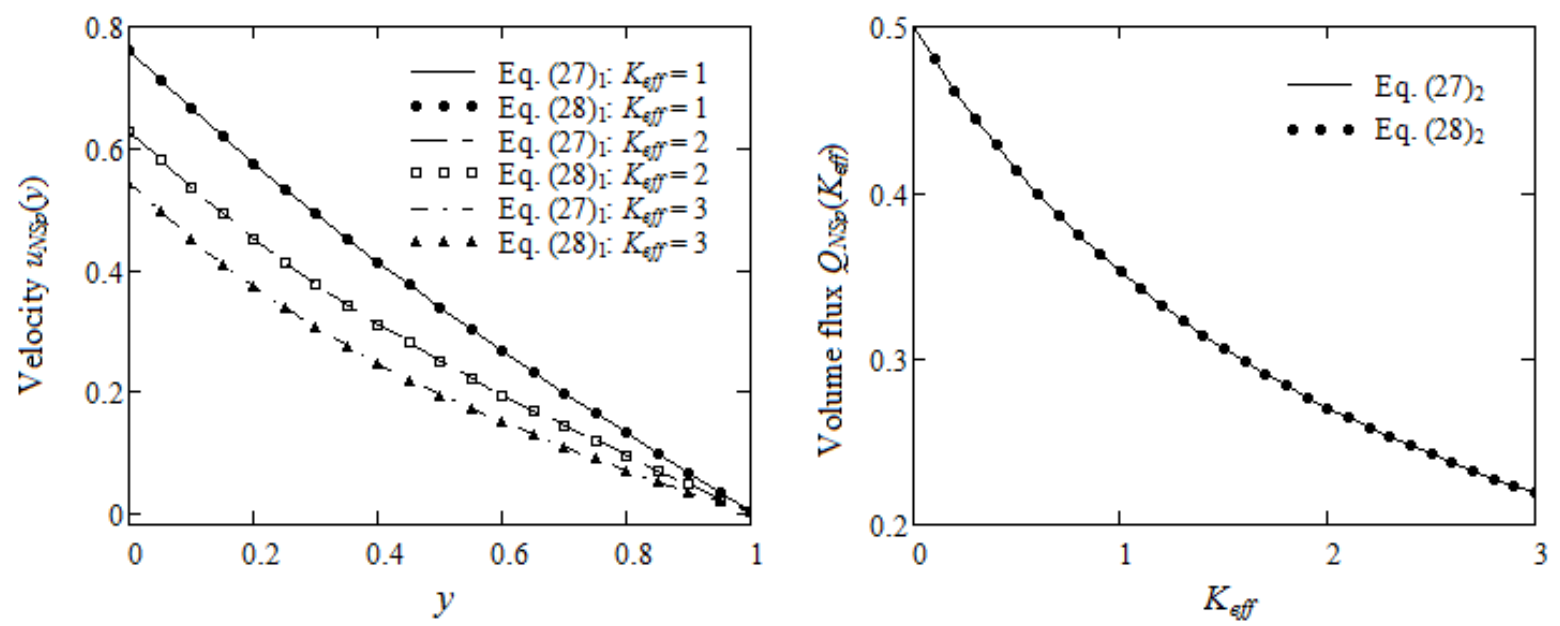

Figure 1. The equivalence of steady solutions given by the relations (27) and (28).
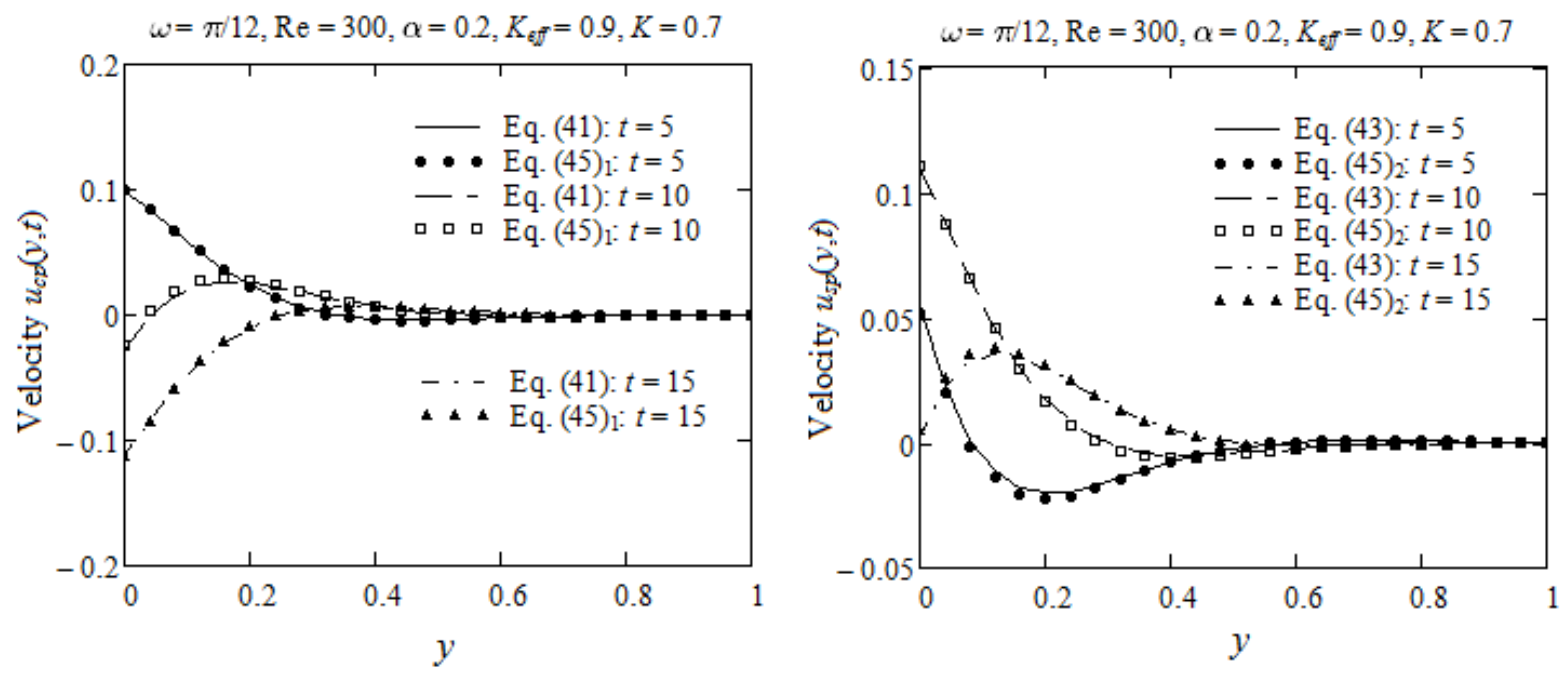

Figure 2. The equivalence of steady-state solutions $u_{c p}(y, t)$ and $u_{s p}(y, t)$ given by the equations (41) and (43) with those from Eq. (45), respectively (45) $)_{2}$.

It was also proved that the solutions corresponding to motions generated by ramp-type shear stresses on the boundary can be immediately determined if the similar solutions for the flow induced by a constant shear stress on the boundary are known.

Figures 3-6 are here included to bring to light the influence of the magnetic field and porous medium on the fluid motion. More precisely, we would like to know if they delay or hasten the appearance of the steady state for motions which 
become steady in time. These graphical representations have been prepared for fixed values of $\alpha=0.5, \omega=\pi / 12$, $\mathrm{Re}=300$ and distinct values of the magnetic and porous parameters $M$, respectively $K$. It is clearly seen from these figures that the necessary time to touch the steady state is a decreasing function with regard to both parameters. Consequently, the steady state for such motions of ISGF is rather obtained in the presence of the magnetic field or porous medium.

In addition, from Figures 3 and 4 as well as from the first Figure 1, it clearly results that the fluid velocity diminishes for increasing values of $M$ or $K$. Consequently, the fluid flows slower in the presence of a magnetic field or a porous medium. This is possible because the presence of a magnetic field induces a resistive force (also called Lorentz force) that leads to deceleration of the flow. On the other hand, if $K$ increases, the resistance of the porous medium also grows and the fluid velocity diminishes. As a result, the volume flux $Q$ is also a decreasing function with regard to these parameters (see the second Figure 2 for the steady motion). In all cases corresponding to the motion due to a constant shear stress on the boundary, the fluid velocity smoothly decreases from maximum values on the lower plate to the zero value on the stationary plate.
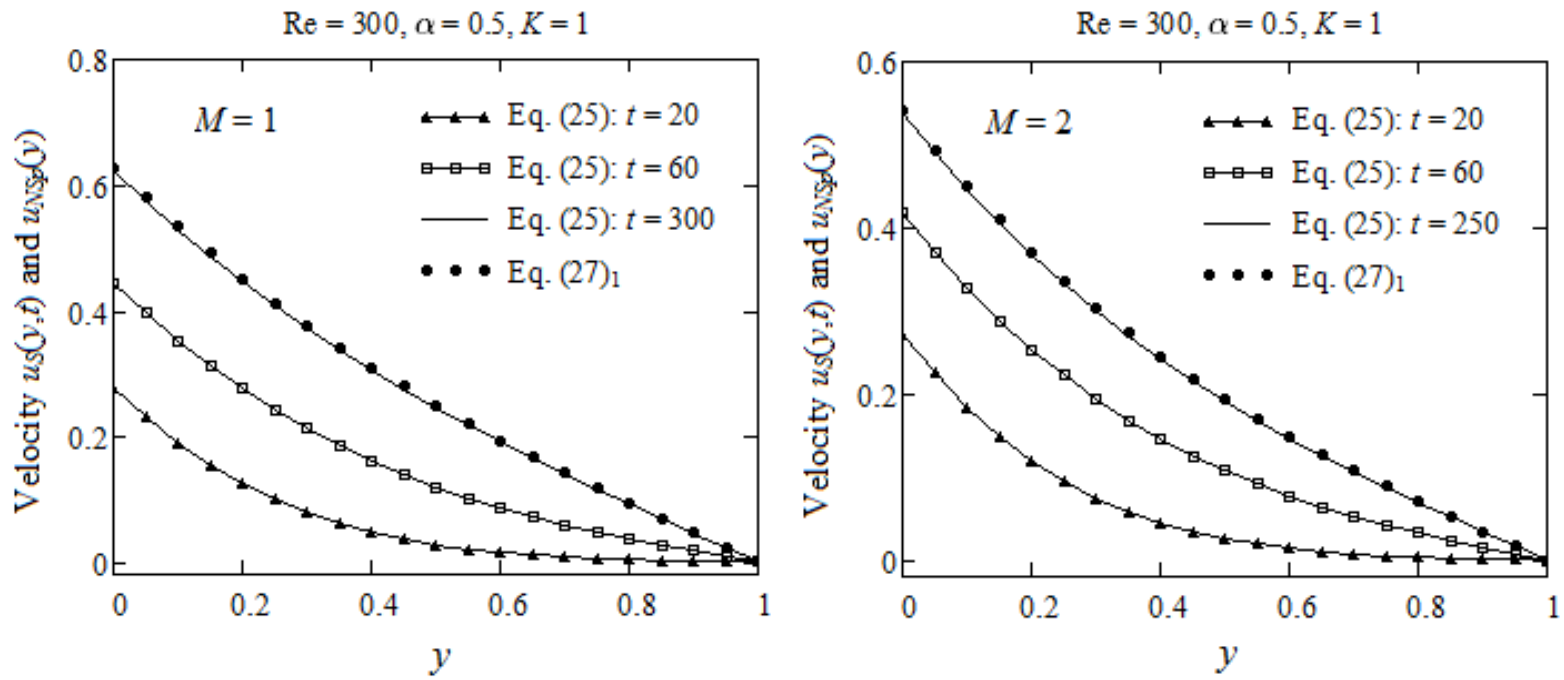

Figure 3. Convergence of starting solution $u_{S}(y, t)$ given by Eq. (25) to its steady component $u_{N S p}(y)$ for two distinct values of the magnetic parameter $M$.
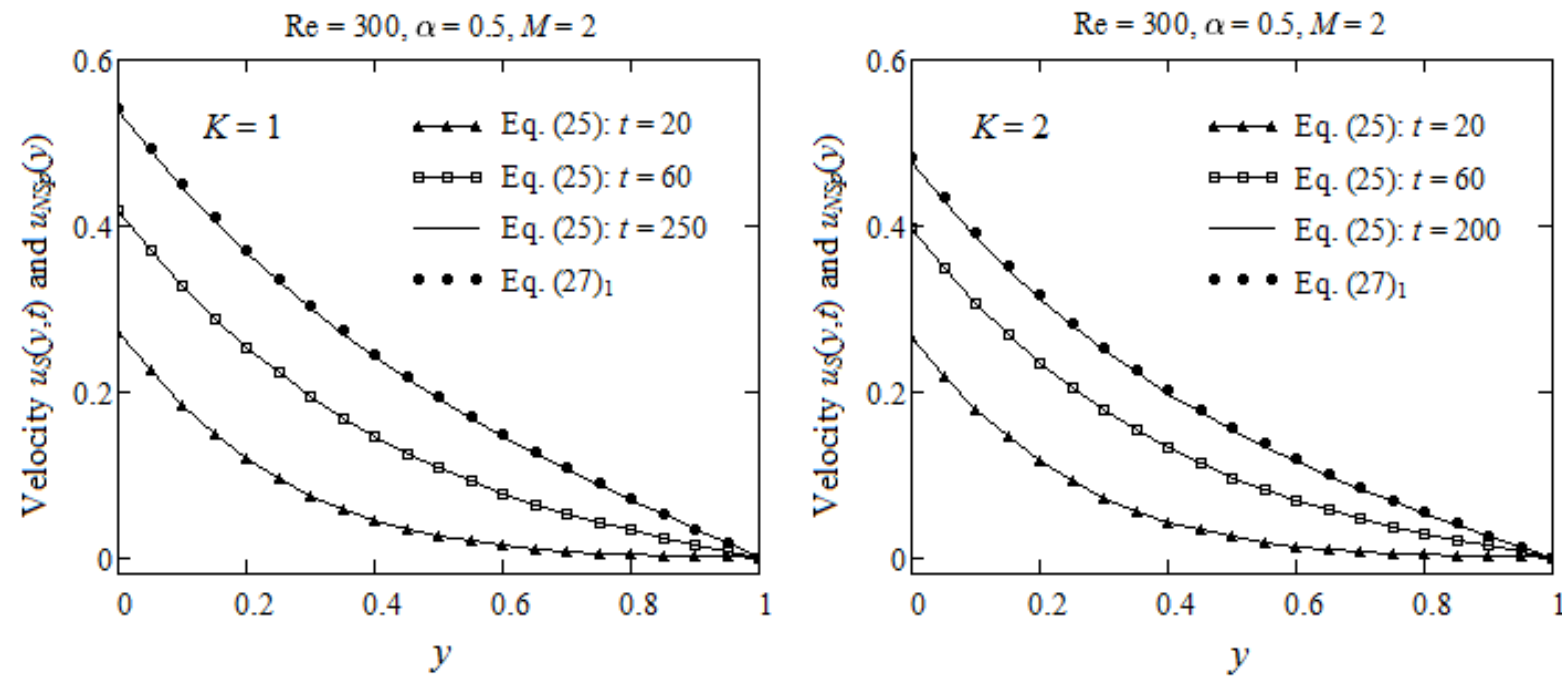

Figure 4. Convergence of starting solution $u_{S}(y, t)$ given by Eq. (25) to its steady component $u_{N S p}(y)$ for two distinct values of the porosity parameter $K$. 

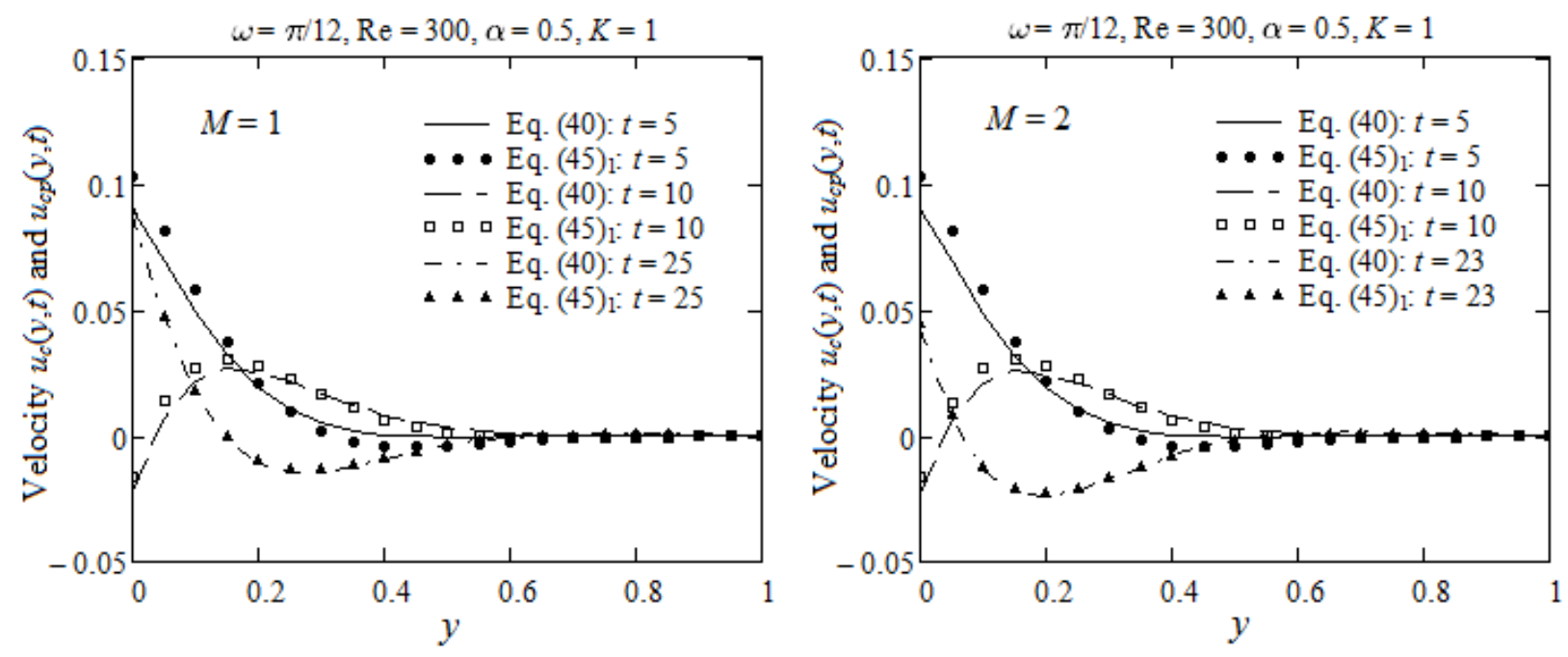

Figure 5. Convergence of starting solution $u_{c}(y, t)$ given by Eq. (40) to its steady state component $u_{c p}(y, t)$ for two distinct values of the magnetic parameter $M$.
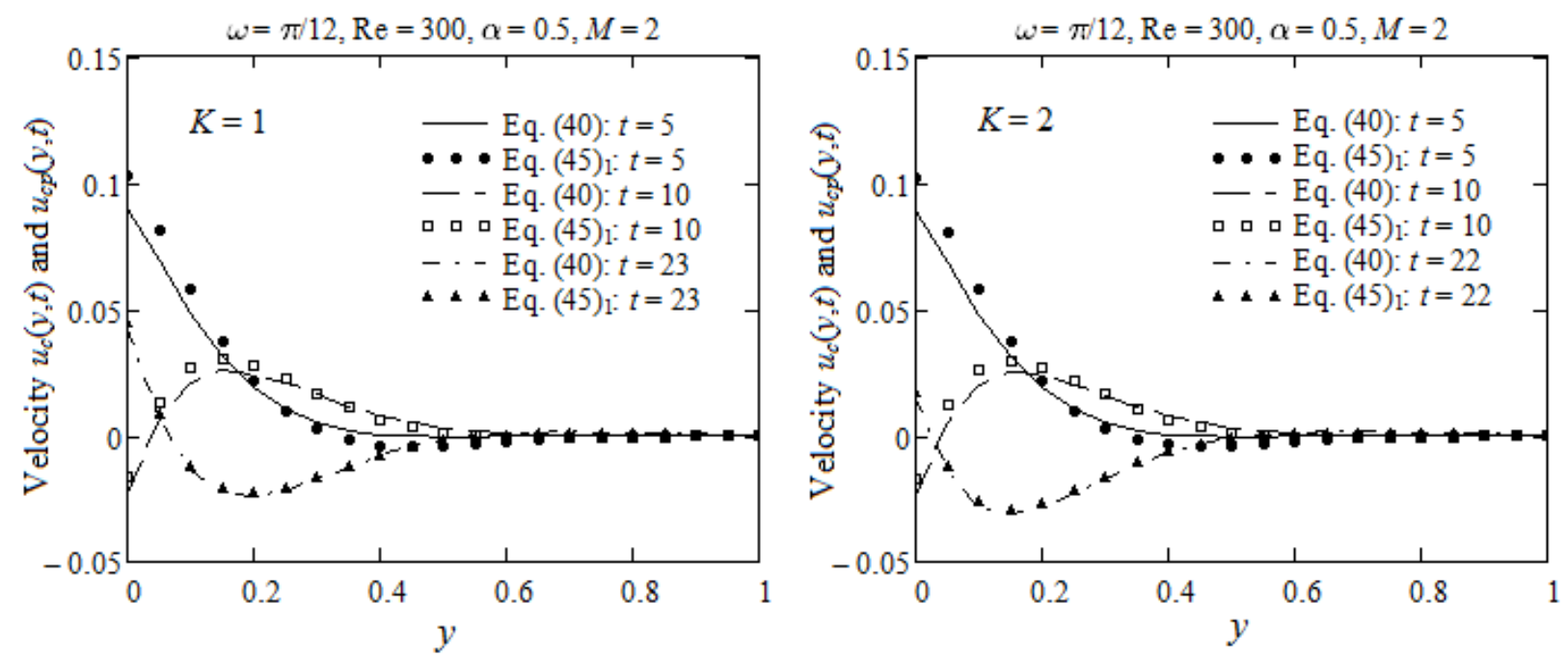

Figure 6. Convergence of starting solution $u_{c}(y, t)$ given by Eq. (40) to its steady state component $u_{c p}(y, t)$ for two distinct values of the porosity parameter $K$.

The main results that have been obtained in this paper are:

- The MHD flow of the ISGF between infinite horizontal parallel plates embedded in a porous medium is completely solved when the lower plate applies a shear stress to the fluid.

- For illustration, the solutions corresponding to three motions with technical relevance are provided. Required time to reach the steady-state for two of them is graphically obtained.

- Steady state for motions which become steady in time is rather obtained in the presence of a magnetic field or porous medium that also determines a reduction of the fluid velocity.

-The solutions for motions induced by ramp-type shear stresses on the boundary can be immediately determined if solutions of the motion due to a constant shear stress are known.

- Permanent components of the solutions corresponding to unsteady motions of the ISGF which become steady in time do not depend of magnetic and porous parameters ( $M$ and $K$ ) independently, but only by a combination of them which is $K_{\text {eff }}=M+K$.

-Starting solutions corresponding to Newtonian fluids performing same motions, as well as the solutions of Stokes' problems [21], also depend of $M$ and $K$ by means of $K_{\text {eff }}$ only. 


\section{Appendix}

$$
\begin{gathered}
u(y, t)=2 \sum_{n=0}^{\infty} u_{F n}(t) \cos \left(\mu_{n} y\right) \text { if } u_{F n}(t)=\int_{0}^{1} u(y, t) \cos \left(\mu_{n} y\right) d y \text { and } \mu_{n}=(2 n+1) \pi / 2, \\
\int_{0}^{1} \frac{\partial^{2} u(y, t)}{\partial y^{2}} \cos \left(\mu_{n} y\right) d y=-\mu_{n}^{2} u_{F n}(t)-\left.\frac{\partial u(y, t)}{\partial y}\right|_{y=0}+(-1)^{n} \mu_{n} u(1, t), \\
2 \sum_{n=0}^{\infty} \frac{\cos \left(\mu_{n} y\right)}{\mu_{n}^{2}}=1-y \text { for } 0 \leq y \leq 1 \text { and } \mu_{n}=(2 n+1) \pi / 2, \\
H^{\prime}(t)=\delta(t) \text { (the Dirac delta function) and } \int_{0}^{t} \delta(t-s) g(s) d s=g(t) . \\
2 \sum_{n=0}^{\infty} \frac{(-1)^{n}}{\mu_{n}^{3}}=\frac{1}{2} \text { for } 0 \leq y \leq 1 \text { and } \mu_{n}=(2 n+1) \pi / 2 .
\end{gathered}
$$

\section{References}

[1] Schlichting, H. (1968). Boundary Layer Theory, McGraw-Hill, New York.

[2] Wang, C. Y. (1989). Exact solutions of the unsteady Navier-Stokes equations, Appl. Mech. Rev., 42(11S), S269-S282. https://doi.org/10.1115/1.3152400.

[3] Erdogan, M. E. (2002). On the unsteady unidirectional flows generated by impulsive motion of a boundary or sudden application of a pressure gradient, Int. J. Non Linear Mech., 37, 1091-1106. https://doi.org/10.1016/S0020-7462(01)00035-X.

[4] Erdogan, M. E. and Imrak, C. E. (2007). On some unsteady flows of a non-Newtonian fluid. Appl. Math. Model., 31, 170-180. https://doi.org/10.1016/j.apm.2005.08.019.

[5] Mendiburu, A. A., Carrocci, L. R., and Carvalho, J. A. (2009). Analytical solution for transient one-dimensional Couette flow considering constant and time-dependent pressure gradients, Eng. térm., 8(2), 92-98. http://dx.doi.org/10.5380/reterm.v8i2.61921.

[6] Singha, K. D. and Choudhary, R. C. (1965). Flow of viscous incompressible fluid between two parallel plates, one in uniform motion and the other at rest, with suction at the stationary plate, Proc. Indian Acad. Sci., 61(5), 308-318.

[7] Kiema, D. W., Manyonge, W. A., Bitok, J. K., Adenyah, R. K., and Barasa, J. S. (2015). On the steady MHD Couette flow between two infinite parallel plates in a uniform transverse magnetic field, J. Appl. Math. Bioinf., 5(1), 87-99. https://repository.maseno.ac.ke/handle/123456789/1785.

[8] Onyango, E. R., Kinyanjui, M. N., and Uppal, S. M. (2015). Unsteady hydromagnetic Couette flow with magnetic field lines fixed relative to the moving upper plate, Am. J. Appl. Math., 3(5), 206-214. doi: 10.11648/j.ajam.20150305.11.

[9] Al-Hadhrami, A. K., Elliot, L., Ingham, D. B., and Wen, X. (2003). Flow through horizontal channels of porous materials, Int. J. Energy Res., 27(10), 875-889. https://doi.org/10.1002/er.923.

[10] Dash, G. C. and Ojha, K. L. (2018). Viscoelastic hydromagnetic-flow between two porous parallel plates in the presence of a sinusoidal pressure gradient. Alex. Eng. J., 57(4), 3463-3471. https://doi.org/10.1016/j.aej.2017.12.011.

[11] Fetecau, C. and Narahari, M. (2020). General solutions for hydromagnetic flow of viscous fluids between horizontal parallel plates through porous medium. J. Eng. Mech., 146(6), 04020053. DOI: 10.1061/(ASCE)EM.1943-7889.0001785.

[12] Javaid, Maria, Imran, M., Fetecau, C., and Vieru, D. (2020). General solutions for the mixed boundary value problem associated to hydromagnetic flows of viscous fluid between symmetrically heated parallel plates. Therm. Sci., 24(2B), 1389-1405. https://doi.org/10.2298/TSCI190608384J.

[13] Ting, T. W. (1965) Certain non-steady flows of second grade fluids. Arch. Ration. Mech. Anal., 14, 1-26. https://doi.org/10.1007/BF00250690. 
[14] Rajagopal, K. R. (1982). A note on unsteady unidirectional flows of a non-Newtonian fluid. Int. J. Non Linear Mech. 17(5/6), 369-373. https://doi.org/10.1016/0020-7462(82)90006-3.

[15] Hayat, T., Khan, I., Ellahi, R., and Fetecau, C. (2008). Some MHD flows of a second grade fluid through the porous medium, Journal of Porous Media, 11(4), 389-400. DOI: 10.1615/JPorMedia.v11.i4.50.

[16] Imran, M., Tahir, M., Nazar, M., and Kamran, M. (2015). Some Couette flows of a second grade fluid due to tangential stresses, Sci. Int., 27(3), 1809-1814.

[17] Ali, F., Khan, M., and Gohar, M. (2021). Magnetohydrodynamic Fluctuating Free Convection Flow of Second-Grade Fluid Flow in a Porous Medium, Math. Probl. Eng., Volume 2021, Article ID 6648281, 13 pages. https://doi.org/10.1155/2021/6648281.

[18] Rivlin, R. S. and Ericksen, J. L. (1955). Stress deformation relation for isotropic materials, J. Ration. Mech. Anal., 4, 323-425.

[19] Dunn, J. E. and Fosdick, R. L. (1974). Thermodynamics, stability and boundedness of fluids of complexity 2 and fluids of second grade, Arch. Ration. Mech. Anal., 56, 191-252. https://doi.org/10.1007/BF00280970.

[20] Cramer, K. R. and Pai, S. I. (1973). Magnetofluid dynamics for engineers and applied physicists. McGraw-Hill, New York.

[21] Fetecau, C., Ellahi, R., Khan, M., and Shah, N. A. (2018). Combined porous and magnetic effects on some fundamental motions of Newtonian fluids over an infinite plate. J. Porous Media, 21(7), 589-605. DOI: 10.1615/JPorMedia.v21.i7.20.

[22] Fetecau, C. and Vieru, D. (2020). Exact solutions for unsteady motion between parallel plates of some fluids with power-law dependence of viscosity on the pressure, Applications in Engineering Science, 1, 100003. https://doi.org/10.1016/j.apples.2020.100003.

[23] Rajagopal, K. R. (2013). A new development and interpretation of the Navier-Stokes fluid which reveals why the Stokes assumption is inapt. Int. J. Non Linear Mech., 50, 141-151. https://doi.org/10.1016/j.ijnonlinmec. 2012.10.007. 